\title{
ON AN EQUIVALENT DEFINITION OF THE TRANSFINITE DIAMETER
}

\author{
PHILIP DAVIS AND HENRY POLLAK
}

Let

$$
f(\zeta)=a \zeta+a_{0}+a_{1} / \zeta+\cdots, \quad a>0,
$$

map the exterior of the unit circle $C$ onto the exterior of a simplyconnected domain $B$. It is part of a general theory (see e.g. [3]) that the quantity $1 / a$ can be identified with the transfinite diameter of $B$. In particular, let $T_{n}(z)$ be the polynomial of degree $n$ with leading coefficient 1 whose $L^{\infty}$ norm over $B$, i.e. the maximum of whose absolute value over $\bar{B}$, is minimum. Then

$$
\lim _{n \rightarrow \infty}\left(M_{n}\right)^{1 / n}=a .
$$

The object of this paper is to show that the last result holds true in the case of domains with a Jordan boundary if we replace the minimal polynomials in the $L^{\infty}$ metric by those in any $L^{p}$ metric, $p \geqq 2$. Let $Q_{n}^{(p)}(z)$ be the polynomial minimizing

$$
\left(\iint_{B}\left|Q_{n}(z)\right|^{p} d x d y\right)^{1 / p}
$$

among all polynomials of degree $n$ with leading coefficient 1 , and let $\lambda_{n}^{(p)}$ be the $L^{p}$ norm of $Q_{n}^{(p)}(z)$, i.e. the value of (2) for $Q_{n}=Q_{n}^{(p)}$. Then $\lim _{n \rightarrow \infty}\left(\lambda_{n}^{(p)}\right)^{1 / n}=a$. After a preliminary lemma substantially due to Carleman [2], we shall prove the result first for $p=2$, and then for $2<p<\infty$.

Lemma. Let $B$ have an analytic boundary. Then $\lim _{n \rightarrow \infty}\left(\lambda_{n}^{(2)}\right)^{1 / n}=a$.

Proof. Since $T_{n}(z)$ is a competing polynomial in the $L^{2}$ minimum problem,

$$
\left(\lambda_{n}^{(2)}\right)^{2} \leqq \iint_{B}\left|T_{n}(z)\right|^{2} d x d y \leqq M_{n}^{2} \cdot A,
$$

where $A$ is the area of $B$, and

$$
\limsup _{n \rightarrow \infty}\left(\lambda_{n}^{(2)}\right)^{1 / n} \leqq \lim \left(M_{n}\right)^{1 / n}=a .
$$

On the other hand, since $B$ has an analytic boundary, the function

Received by the editors May 11, 1951. 
$f(\zeta)$ can be continued to be analytic and schlicht up to a circle $\gamma_{\rho_{1}}:|\zeta|$ $=\rho_{1}<1$. Let $\rho_{1}<\rho<1$ and let $D$ be the annulus bounded by $\gamma_{\rho}$ and $\gamma_{1}$; furthermore, let $f(D)$ be its image in $B$. Then

$$
\begin{aligned}
\iint_{B}\left|Q_{n}^{(2)}(z)\right|^{2} d x d y & \geqq \iint_{f(D)}\left|Q_{n}^{(2)}(z)\right|^{2} d x d y \\
& =\iint_{D}\left|Q_{n}^{(2)}[f(\zeta)] f^{\prime}(\zeta)\right|^{2} d \xi d \eta .
\end{aligned}
$$

Now $Q_{n}^{(2)}(z)=z^{n}+c_{1} z^{n-1}+\cdots, f(\zeta)=a \zeta+a_{0}+a_{1} / \zeta+\cdots ;$ hence

$$
Q_{n}^{(2)}[f(\zeta)] f^{\prime}(\zeta)=a^{n+1} \zeta^{n}+\sum_{j=1}^{\infty} b_{i} \zeta^{n-i}
$$

and

$$
\begin{aligned}
\left(\lambda_{n}^{(2)}\right)^{2} & \geqq \iint_{D}\left|a^{n+1} \zeta^{n}+\sum_{j=1}^{\infty} b_{j} \zeta^{n-j}\right|^{2} d \xi d \eta \\
& =2 \pi a^{2 n+2} \int_{\rho}^{1} r^{2 n+1} d r+2 \pi \sum_{j=1}^{\infty}\left|b_{j}\right|^{2} \int_{\rho}^{1} r^{2 n-2 j+1} d r \\
& \geqq \frac{\pi a^{2 n+2}}{n+1}\left(1-\rho^{2 n+2}\right) .
\end{aligned}
$$

Since $\rho<1$, it follows that $\rho^{2 n+2} \rightarrow 0$, and $\lim \inf _{n \rightarrow \infty}\left(\lambda_{n}^{(2)}\right)^{1 / n} \geqq a$. By comparing (4) and (6) we see that $\lim _{n \rightarrow \infty}\left(\lambda_{n}^{(2)}\right)^{1 / n}$ exists and equals $a$.

THEOREM 1. Let $B$ be a simply-connected domain with a Jordan boundary. Then $\lim _{n \rightarrow \infty}\left(\lambda_{n}^{(2)}\right)^{1 / n}$ exists and equals a.!

PROof. Let $B \supset B^{\prime}$. Then if $Q_{n}^{(2)}(z)$ is the $L^{2}$ minimal polynomial over $B$,

(7) $\left[\lambda_{n}^{(2)}(B)\right]^{2}=\iint_{B}\left|Q_{n}(z)\right|^{2} d x d y \geqq \iint_{B^{\prime}}\left|Q_{n}(z)\right|^{2} d x d y \geqq\left[\lambda_{n}^{(2)}\left(B^{\prime}\right)\right]^{2}$.

Hence

$$
\limsup _{n \rightarrow \infty}\left[\lambda_{n}^{(2)}(B)\right]^{1 / n} \geqq \limsup _{n \rightarrow \infty}\left[\lambda_{n}^{(2)}\left(B^{\prime}\right)\right]^{1 / n} ;
$$

the same is true for lim inf, and lim if it exists. Thus, we see that $\lim \sup _{n \rightarrow \infty}\left[\lambda_{n}^{(2)}(B)\right]^{1 / n}$ and $\lim \inf _{n \rightarrow \infty}\left[\lambda_{n}^{(2)}(B)\right]^{1 / n}$ are increasing set functions. Now an arbitrary domain can be approximated from the exterior by domains with analytic boundaries in such a way that the 
respective $a(B)$ 's are arbitrarily close to each other; one needs only to take level lines of the exterior mapping function. In the case of a domain with a Jordan boundary, it follows from the Caratheodory theory [1] that this is also true for interior approximation. Since the transfinite diameter is also an increasing set function, it follows by approximation that $\lim \left(\lambda_{n}^{(2)}\right)^{1 / n}$ exists and equals $a$ in the case of an arbitrary Jordan domain.

TheOREM 2. Let $Q_{n}^{(p)}(z)$ be the polynomial of degree $n$ with leading coefficient 1 minimizing

$$
\left(\iint_{B}\left|Q_{n}(z)\right|^{p} d x d y\right)^{1 / p}
$$

and let $\lambda_{n}^{(p)}$ be the corresponding minimum value of (9). Then for any $p>2, \lim _{n \rightarrow \infty}\left(\lambda_{n}^{(p)}\right)^{1 / n}$ exists and equals $a$.

Proof. For any $f(z) \in L^{2}$ and $L^{p}, p>2$, we have

$$
\left(\iint_{B}|f(z)|^{2} d x d y\right)^{1 / 2} \leqq\left(\iint_{B}|f(z)|^{p} d x d y\right)^{1 / p} .
$$

Therefore

$$
\lambda_{n}^{(2)} \leqq\left(\iint_{B}\left|Q_{n}^{(p)}(z)\right|^{2} d x d y\right)^{1 / 2} \leqq\left(\iint_{B}\left|Q_{n}^{(p)}(z)\right|^{p} d x d y\right)^{1 / p}=\lambda_{n}^{(p)}
$$

and hence

$$
a=\lim _{n \rightarrow \infty}\left(\lambda_{n}^{(2)}\right)^{1 / n} \leqq \liminf _{n \rightarrow \infty}\left(\lambda_{n}^{(p)}\right)^{1 / n}
$$

On the other hand,

$$
\left(\lambda_{n}^{(p)}\right)^{p} \leqq \iint_{B}\left|T_{n}(z)\right|^{p} d x d y \leqq A\left[M_{n}\right]^{p}
$$

where $A$ is the area of $B$. Hence

$$
\limsup _{n \rightarrow \infty}\left(\lambda_{n}^{(p)}\right)^{1 / n} \leqq \lim \left(M_{n}\right)^{1 / n}=a
$$

Combining (11) and (12), we obtain that $\lim _{n \rightarrow \infty}\left(\lambda_{n}^{(p)}\right)^{1 / n}$ exists and equals $a$.

\section{BIBLIOGRAPHY}

1. C. Carathéodory, Untersuchungen ueber die konformen Abbildungen von festen 
und veraenderlichen Gebieten, Math. Ann. vol. 72 (1912) pp. 107-144.

2. T. Carleman, Ueber die Approximation analytischer Funktionen durch lineare Aggregate von vorgegebenen Potensen, Archiv foer Mathematik, Astronomi och Fysik vol. 17 (1922).

3. G. P6lya and G. Szegö, Ueber den transfiniten Durchmesser von ebenen und raeumlichen Punktmengen, J. Reine Angew. Math. vol. 165 (1931).

HARVARD UNIVERSITY 